\title{
OTIMIZAÇÃO DO PLANO DE PASSES DO LAMINADOR DE AÇOS LONGOS DA CSN*
}

Fabricio Reis de Almeida ${ }^{1}$ Yuri Daboit Nardelli²

Giovane Naves do Amara/ ${ }^{3}$

\section{Resumo}

O presente trabalho tem como objetivo mostrar os ganhos financeiros e produtivos do Laminador de Aços Longos da CSN através da otimização do plano de passes. Analisando a árvore de calibração identificamos a possibilidade de ganhos com a unificação de famílias de bitolas. No laminador temos duas famílias: Família $\varnothing 5,5$ mm (bitolas 5,5; 7,0; 9,0; 11,0; 14,30) e Família $\varnothing 6,3 \mathrm{~mm}$ (bitolas 6,3; 8; 10; 12,5 e 16) para a fabricação destas bitolas utilizamos até 18 passes no laminador duo horizontal/vertical e mais ate 10 passes no laminador acabador cantilever. Após um estudo detalhado do plano de passes existente, verificou-se a possibilidade da eliminação e unificação de passes entre as duas famílias. Para corroborar as alterações propostas, decidiu-se utilizar um modelo matemático que representasse os passes e realizasse os cálculos para verificar as reduções e capacidades dos equipamentos visando minimizar os riscos e custos adicionais com modificações de ferramental. Após mais de 60 horas de simulações e testes, foi desenhado um novo plano de passes para a Laminação eliminando-se a necessidade de troca de 8 cadeiras, o que representa um aumento de 6 horas no tempo disponível para produção por mês e também a redução de 8 pares de cilindros de laminação, redução de guias e roletes, e tempo de preparação na oficina de cilindros. Só após verificação e aprovação desta etapa foram iniciados os testes no laminador que confirmaram os resultados obtidos nas simulações.

Palavras-chave: Plano de passes, Calibração, Laminação aços longos.

\section{Abstract}

\section{OPTIMIZATION OF PASSES PLAN OF CSN'S ROLLING MILL}

This present paper shows the financial and productivity gains of CSN's rolling mill through the pass plan optimization. Analyzing the pass design we identified the possibility of gains from the unification of sizes of families. The mill has two families: family $\varnothing 5,5 \mathrm{~mm}$ ( gauges $-5,5 ; 7,0 ; 9,0 ; 11,0 ; 14,30$ ) and family $\varnothing 6,35 \mathrm{~mm}$ ( gauges $6,35 ; 8,0 ; 10 ; 12,5 ; 16,0)$ for manufacturing these gauges it is used up to 10 passes in horizontal/vertical duo mill and other more up to 10 passes through the mill finisher cantilever. After a deep study of the existing plan passes, there was the possibility of elimination and unification of passes between the two families. In order support the proposed amendments, it was decided to use a mathematical model that represented the passes and perform calculations to verify the reductions of capacity and equipment to minimize the risks and additional costs for tooling changes. After more than 60 hours of testing and simulations, a new pass was designed for the pass plan eliminating the need to exchange 8 stands, which represents an increase of 6 hours on the available time for production per month and also the reduction of 8 pairs of rolls, numbers of rollers and guides, and setup time in the roll shop. Only after verification and approval of the this stage the tests were started in the mill which confirmed the results obtained in the simulations.

Keywords: Passes plan; Pass design; Rolling mill.

1 Engenheiro metalúrgico, Engenheiro Especialista, CSN - Companhia Siderúrgica Nacional, Volta Redonda, Rio de Janeiro, Brasil.

2 Engenheiro Metalúrgico, Gerente Técnico, CSN, Volta Redonda, Rio de Janeiro, Brasil.

3 Projetista, CSN, Volta Redonda, Rio de Janeiro, Brasil. 


\section{INTRODUÇÃO}

O estudo de passes ou deformações de um laminador de aços longos é conhecido como calibração. O termo calibração é utilizado de forma simplificada para designar o projeto de passes utilizado para executar a laminação de produtos longos. $\mathrm{Na}$ calibração é efetuada a distribuição da deformação total desejada no material em um conjunto de deformações intermediarias ate que se obtenha o produto final desejado. Uma série de deformações intermediarias é denominada de sequência de passes. Cada passe é representado por um canal, cuja a forma, dimensão e longo da mesa de um cilindro de laminação e nas diversas cadeiras que constituem o trem de laminação são definidas no projeto de calibração.

A realização do projeto de calibração para a laminação de um determinado produto longo deve aliar conhecimentos teóricos e práticos acumulados pelo profissional responsável por esta tarefa, normalmente denominado de projetista ou calibrador. Dentre os conhecimentos teóricos necessários podem ser destacados:

- Os mecanismos de deformação, resistência e amaciamento dos aços;

- A distribuição de tensões, deformações e deslocamentos ao longo de uma seção de um produto laminado e ao longo do seu comprimento;

- A variação da temperatura na seção e no comprimento do esboço e ao longo do tempo ou deslocamento do esboço ao percorrer a extensão do trem de laminação;

- O valor e a variação do coeficiente de atrito na interface cilindro/esboço;

- A variação da microestrutura do aço durante a deformação e resfriamento do esboço e;

- noções de custo dos diversos insumos, utilidades e serviços empregados em uma linha de laminação[1].

\subsection{Sequência de Passes}

As sequência de passes combinam os diversos tipos de canais para a atender às variáveis: resistência dos cilindros, ângulos de mordida e reduções suportáveis pelo aço. Como regra geral não devemos praticar reduções superiores a $35 \%$ em área em um passe de laminação. Faixas de valores recomendadas para redução em área praticada:

Tabela 1 - Exemplo de faixas de redução [2]

\begin{tabular}{ll}
\hline Tipo de passe & Redução \\
\hline Quadrado - oval - quadrado & 20 a $28 \%$ \\
Quadrado - oval sueco - quadrado & 25 a $35 \%$ \\
Caixa- caixa & 16 a $24 \%$ \\
Diamante - Diamante & 22 a $30 \%$ \\
Quadrado - diamante - quadrado & 20 a $28 \%$ \\
Redondo - oval - redondo & 12 a $28 \%$ \\
Passe canteador para chatos & 2 a $14 \%$ \\
\hline
\end{tabular}

\subsection{Arvore de Calibração}

A árvore de calibração é um diagrama na forma de uma matriz apresentando todos os passes necessários para a produção de um produto indicando a cadeira em que e laminando cada passe. Assim temos um modelo do tipo matriz onde as linhas são as cadeiras do laminador, as colunas são os produtos do programa de produção, e 
os elementos em cada campo linha-coluna são os passes. Desta forma é possível em um único documento ter a visão geral de todo o processo de passes e deformação realizado no laminador para o seu programa de produção. Na figura 1 apresenta-se um exemplo de uma arvore de calibração para barras redondas[1].

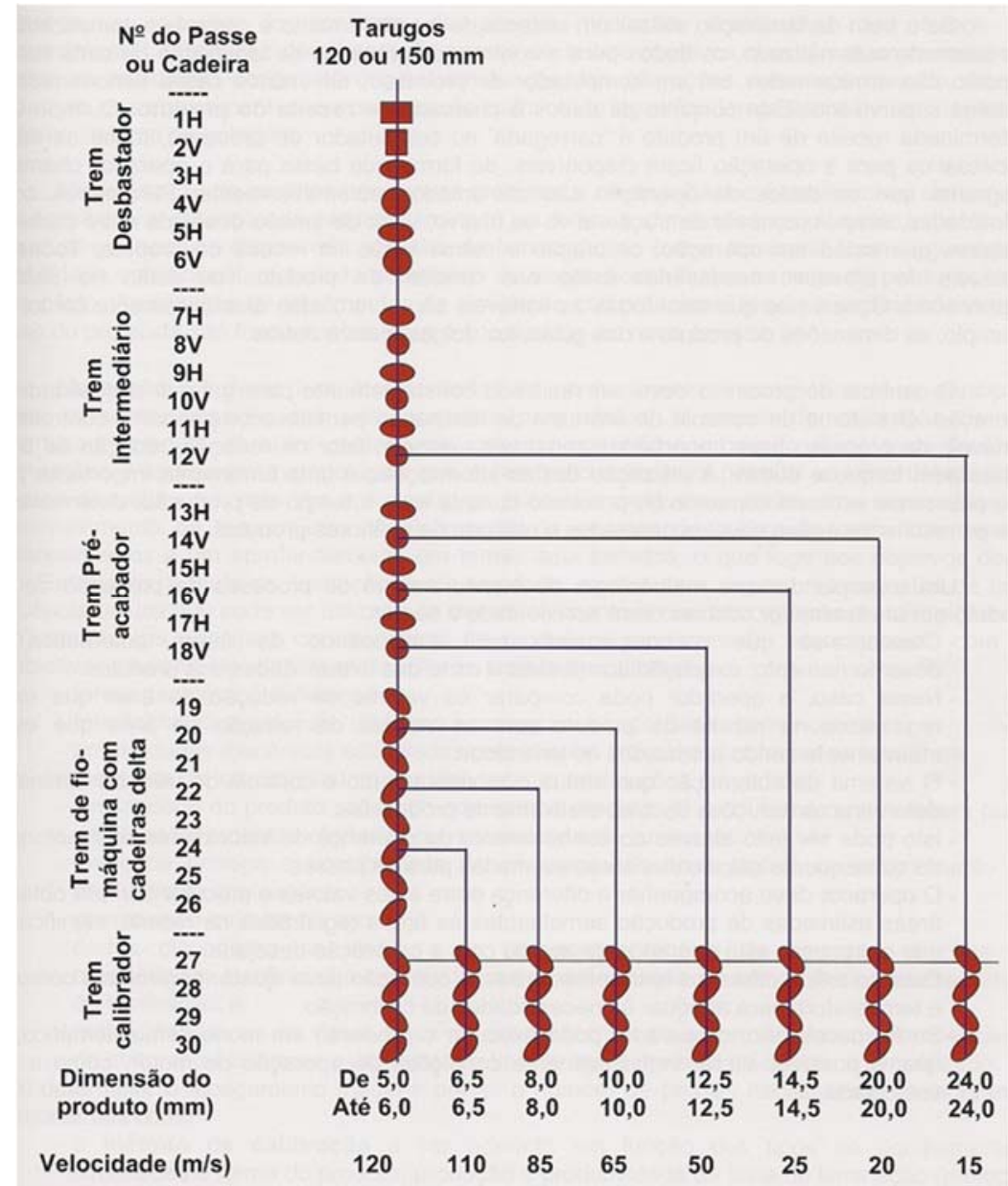

Figura 1 - Exemplo de uma árvore de calibração para barras redondas[1]

\subsection{Projeto de Calibração}

No passado, a realização da calibração era efetuada essencialmente por um projetista com larga experiência no processo de laminação, que se baseava quase que exclusivamente em conhecimentos práticos, mas utilizando sempre que possível em função da metodologia selecionada e dos recursos disponíveis, determinados princípios da mecânica dos materiais. Em determinadas situações, são utilizados simulações em escala laboratorial, utilizando o próprio aço ou, materiais alternativos como chumbo ou plasticilina para realizar simulações físicas de uma determinada sequencia de passes, verificando principalmente o preenchimento dos canais, o fluxo do material e o aparecimento de defeitos superficiais ou internos no material laminado.

Com o advento e disseminação dos computadores, a utilização de programas computacionais contribui sobremaneira para facilitar os cálculos e desenhos envolvidos nas diversas etapas do projeto de canais. Entretanto, a experiência, o bom senso e o talento sempre devem estar presentes quando se trabalha com 
calibração, de modo a permitir a realização de um projeto que possibilite a geração de produtos laminados de qualidade, com boa produtividade e segurança operacional, tanto para equipamentos quanto para o pessoal envolvido diretamente ou indiretamente como controle, inspeção e manutenção do trem de laminação.

Os projetos de laminação do laminador de aços longos da CSN utiliza um software de simulação, este modelo permite que possamos fazer a calibração de todos os passes de laminação, com resultados realistas em relação as velocidades, reduções, temperaturas, torque nos motores, e preenchimento de canais.

\section{MATERIAIS E MÉTODOS}

\subsection{Materiais}

Conforme mencionado acima utilizamos um software que através de um modelo matemático realização as simulações da calibração antes de realizar os testes na linha, o que nos permite operacionalizar as alternativas ou calibração em um menor espaço de tempo e com maior acertividade.

A validação das simulações foi realizada no laminador de Aços longos e em escala industrial.

A fig. 2 mostra a tela do supervisório com o layout da laminação, e disposição dos equipamentos. Assim podemos ver a sequencia em que estes aparecem.

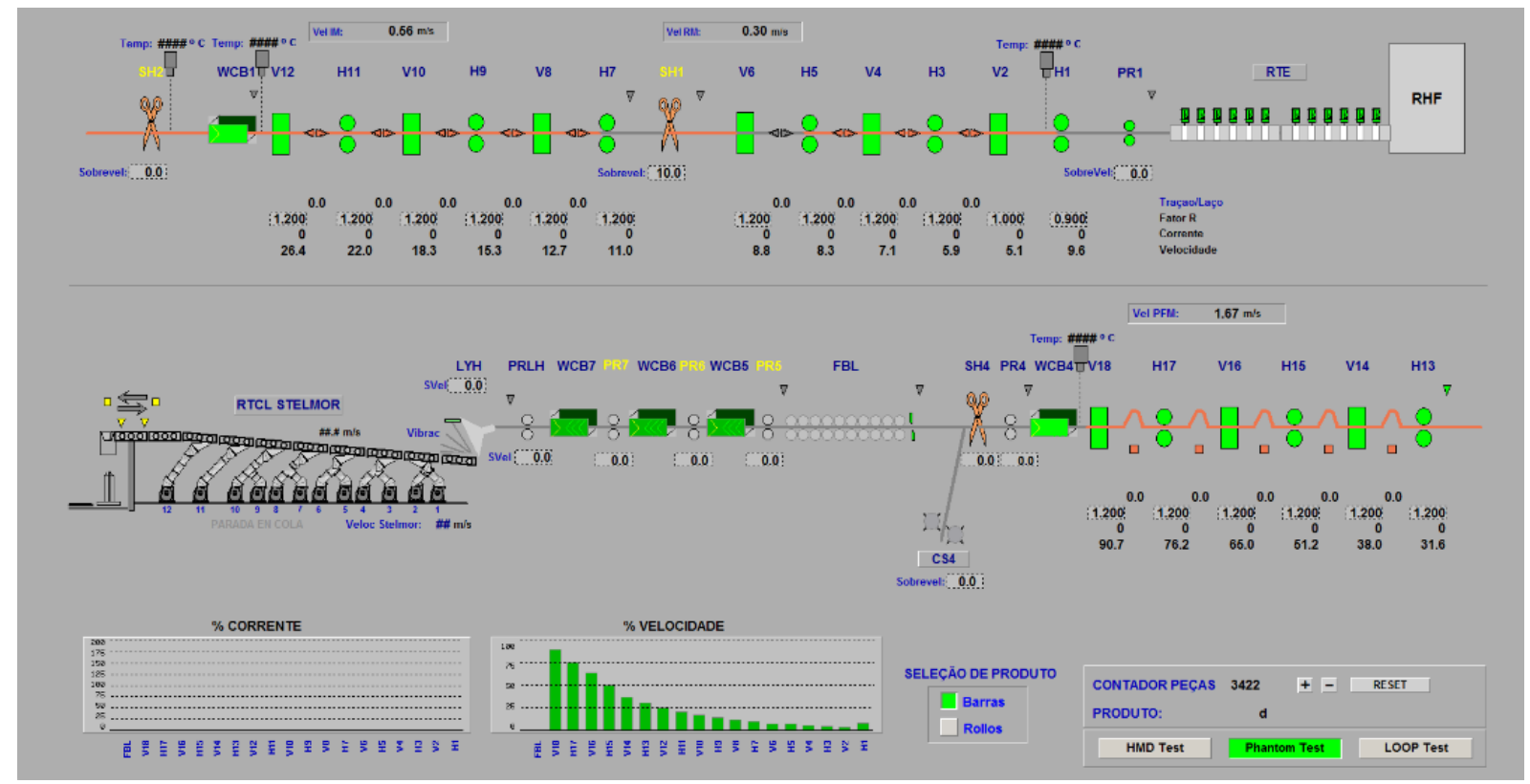

Figura 2 - Imagem da tela do supervisório.

\subsection{Métodos}

\subsubsection{Analise da arvore de calibração}

Foi realizada uma análise detalhada da arvore de calibração original recebida do fornecedor com todos os canais e as sequências de passes. 
$150 \times 150$

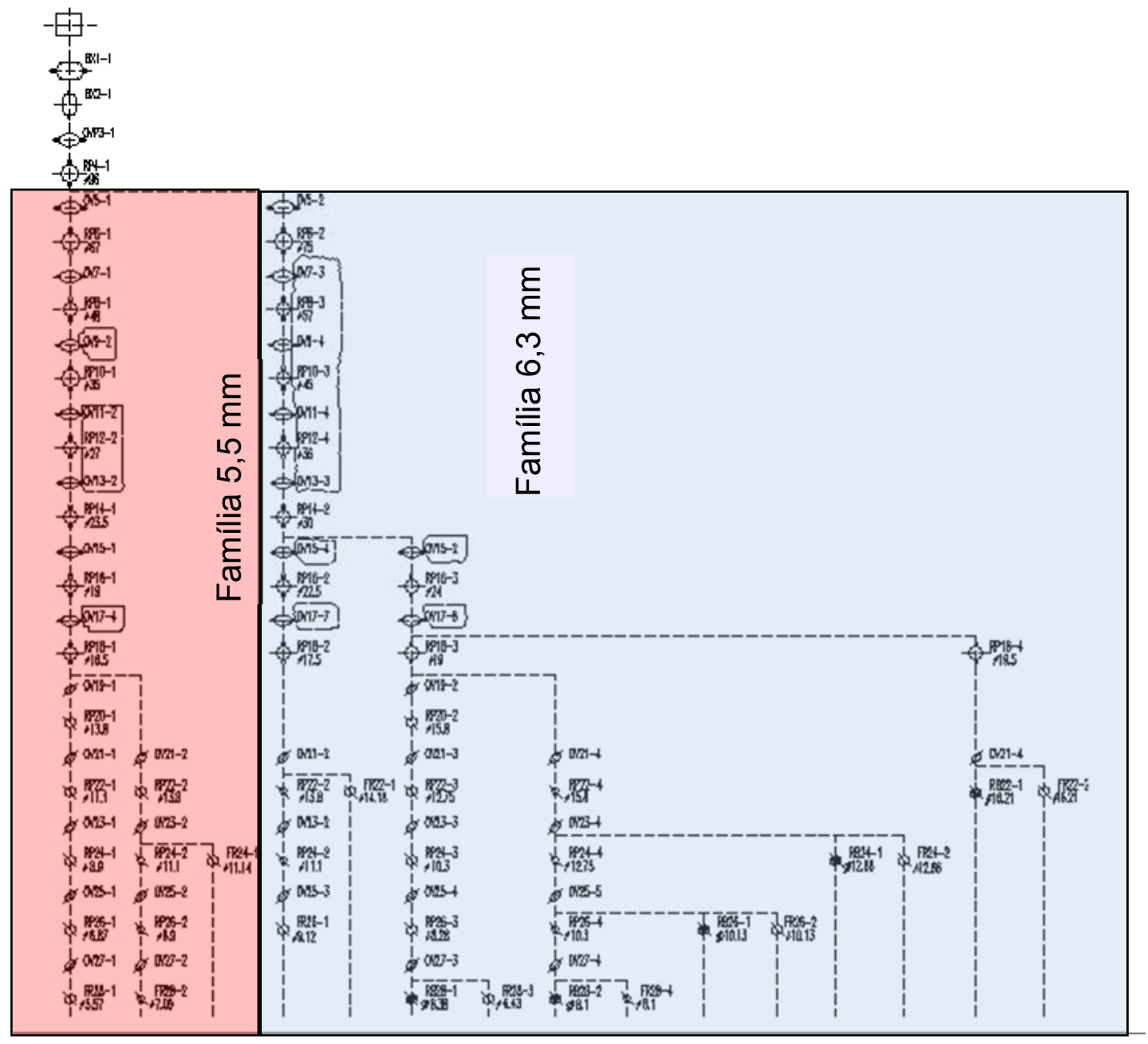

Fonte: CISD

Figura 3. Arvore de calibração.

Como podemos ver na figura 2 a mudança de família sugerida pelo fornecedor aconteceria a partir da cadeira \#5 em diante, isto é, toda troca de família teríamos que trocar ate 24 cadeiras de laminação, incluindo as cadeiras do bloco acabador. Com o tempo médio de 6 horas, considerando que os cilindros de laminação só teriam canais dedicados. Após uma analise verificamos que haviam passes redondos em posições diferentes, como o $\varnothing 35$ da cadeira 10 na família d0 5,5 e o $\varnothing 36$ na cadeira 12 na família do 6,35.

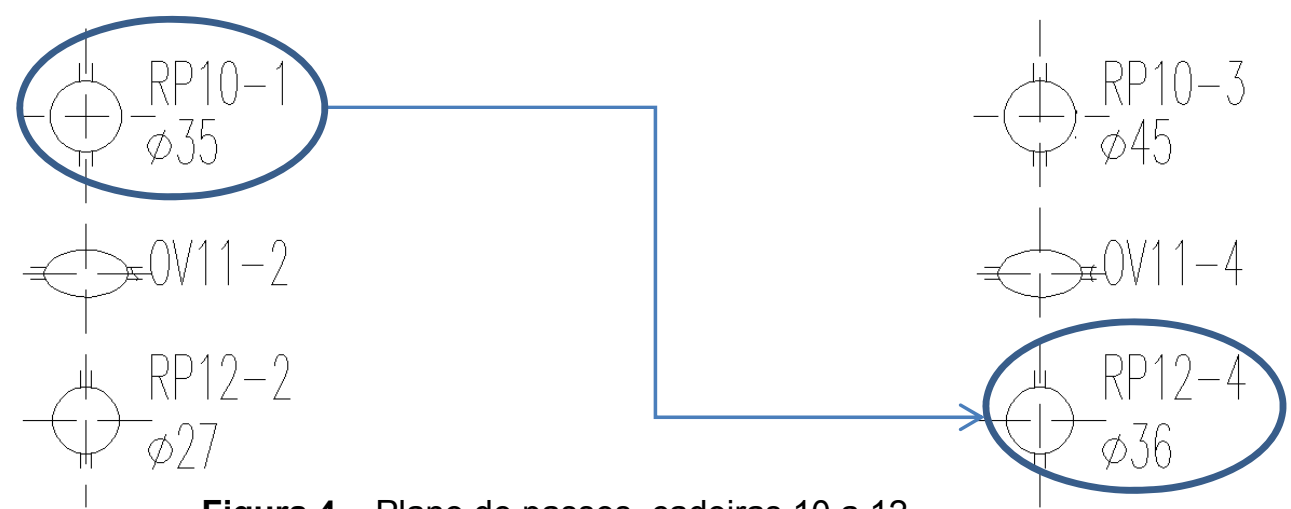

Figura 4 - Plano de passes, cadeiras 10 a 12. 


\subsubsection{Simulações}

Após a analise da arvore de calibração passamos para a segunda etapa que são as simulações:

\begin{tabular}{|c|c|c|c|c|c|c|c|c|c|c|}
\hline PASSE/SEQUÊNCIA & Gaiola 1 & Gaiola 2 & Gaiola 3 & Gaiola 4 & Gaiola 5 & Gaiola 6 & Gaiola 7 & Gaiola 8 & Gaiola 9 & Gaiola 10 \\
\hline Gaiola & $1-1 \mathrm{H}$ & $2-2 \mathrm{~V}$ & $3-3 \mathrm{H}$ & $4-4 \mathrm{~V}$ & $5-5 \mathrm{H}$ & $6-6 \mathrm{~V}$ & $7-7 \mathrm{H}$ & $8-8 \mathrm{~V}$ & $9-9 \mathrm{H}$ & $10-10 \mathrm{~V}$ \\
\hline CANAL & TBX & $\overline{B X}$ & $\overline{\mathrm{BO}}$ & $\mathrm{RD}$ & OV & $\mathrm{RD}$ & OV & $\mathrm{RD}$ & OV & $\mathrm{RD}$ \\
\hline Billet: S0154.3 & BX1-1 & $B \times 2-1$ & OVP3-1 & RP4-1 & OV5-1 & RP6-1 & OV7-1 & RP8-1 & OV9-1 & RP10-1 \\
\hline GAP do Cilindro (vazio) & & & & & & & & & & \\
\hline GAP do Cilindro (ativo) & 19 & 20 & 12 & 11 & 5 & 9 & 7 & 7 & 6 & 8 \\
\hline GAP anterior & & & & & & & & & & \\
\hline GAP DIFF & & & & & & & & & & \\
\hline ALTURA DA BARRA $\mathrm{Mb}$ & 113 & 114 & 76 & 95 & 51,5 & 68 & 36 & 50 & 28,5 & 38,5 \\
\hline LARGURA DA BARRA WB quente & 167 & 127,6 & 143,5 & 90,8 & 114,5 & 66,56 & 83,01 & 47,26 & 60,97 & 36,6 \\
\hline Inscr. Circle Si & & & & & & & & & & \\
\hline REDUÇÃO & 23,44 & 22,85 & 34,98 & 23,42 & 33,48 & 22,85 & 32,2 & 21,71 & 26,28 & 20,94 \\
\hline DIÂMETRO DO CILÍNDRO & 610 & 610 & 610 & 610 & 610 & 610 & 450 & 450 & 450 & 450 \\
\hline DIÂMETRO EFETIVO & 583,88 & 573,37 & 642,7 & 600,5 & 644,79 & 615,9 & 475,98 & 451,48 & 471,04 & 461,03 \\
\hline ROTAÇÂO DO MOTOR & 426 & 562 & 771 & 792 & 838 & 842 & 911 & 932 & 938 & 957 \\
\hline VELOCIDADE & 0,24 & 0,31 & 0,48 & 0,63 & 0,94 & 1,22 & 1,8 & 2,3 & 3,12 & 3,95 \\
\hline Loop/Pull MIN & $-1,00 \%$ & $-1,00 \%$ & $-1,00 \%$ & $-1,00 \%$ & $-1,00 \%$ & $-1,00 \%$ & $-1,00 \%$ & $-1,00 \%$ & $-1,00 \%$ & \\
\hline Loop/Pull CAL. & $-1,00 \%$ & $-1,00 \%$ & $-1,00 \%$ & $-1,00 \%$ & $-1,00 \%$ & $-1,00 \%$ & $-1,00 \%$ & $-1,00 \%$ & $-1,00 \%$ & \\
\hline Loop/Pull MAX & & & & & & & & & & \\
\hline TEMP. DESENFORNAMENTO & 1100 & 1074 & 1054 & 1041 & 1031 & 1028 & 1016 & 1022 & 1026 & 1032 \\
\hline CARGA & 1384 & 1246 & 1697 & 1000 & 1488 & 829 & 934 & 493 & 595 & 349 \\
\hline TORQUE & 132 & 126 & 165 & 92,1 & 126 & 74 & 58,5 & 32,8 & 30 & 19,4 \\
\hline POTÊNCIA & 109 & 138 & 247 & 192 & 369 & 294 & 443 & 335 & 398 & 332 \\
\hline POTÊNCIA MÁX & 149 & 178 & 287 & 232 & 419 & 344 & 493 & 385 & 448 & 382 \\
\hline POTÊNCIA MÉDIA & 143 & 171 & 275 & 223 & 402 & 330 & 473 & 369 & 430 & 366 \\
\hline POTÊNCIA DISPONÍVEL & 284 & 375 & 400 & 400 & 500 & 500 & 500 & 500 & 500 & 500 \\
\hline ÂNGULO DE MORDIDA & 23,3 & 24,8 & 22,5 & 24,8 & 21,5 & 23,7 & 22 & 23,2 & 17 & 18,8 \\
\hline ÁREA DA BARRA QUENTE & 18168 & 14018 & 9114 & 6980 & 4643 & 3582 & 2429 & 1901 & 1402 & 1108 \\
\hline COMP. DA BARRA QUENTE & 15,33 & 19,86 & 30,53 & 39,85 & 59,9 & 77,63 & 114,5 & 146,2 & 198,4 & 251 \\
\hline Spread Coefficient: & 1,1 & 1,1 & 1,2 & 1,2 & 1,3 & 1,2 & 1,3 & 1,2 & 1,3 & 1,2 \\
\hline
\end{tabular}

Figura 5 - Tabela de dados da simulação.

Obtemos assim os redondos necessários para entrada do bloco, um redondo de 19,10 para a família do 5,5 e 17,10 para a família de 5,5 . Verificamos através do modelo que realmente seria possível a obtenção destas bitolas realizando a troca a partir da cadeira 10. Conforme o desenho esquemático abaixo, figura 6 , onde a parte laranja seria a necessidade de troca de cadeiras para o plano de passes antigo, e a parte azul no plano atual e a parte vermelha seria somente retirada das cadeiras.
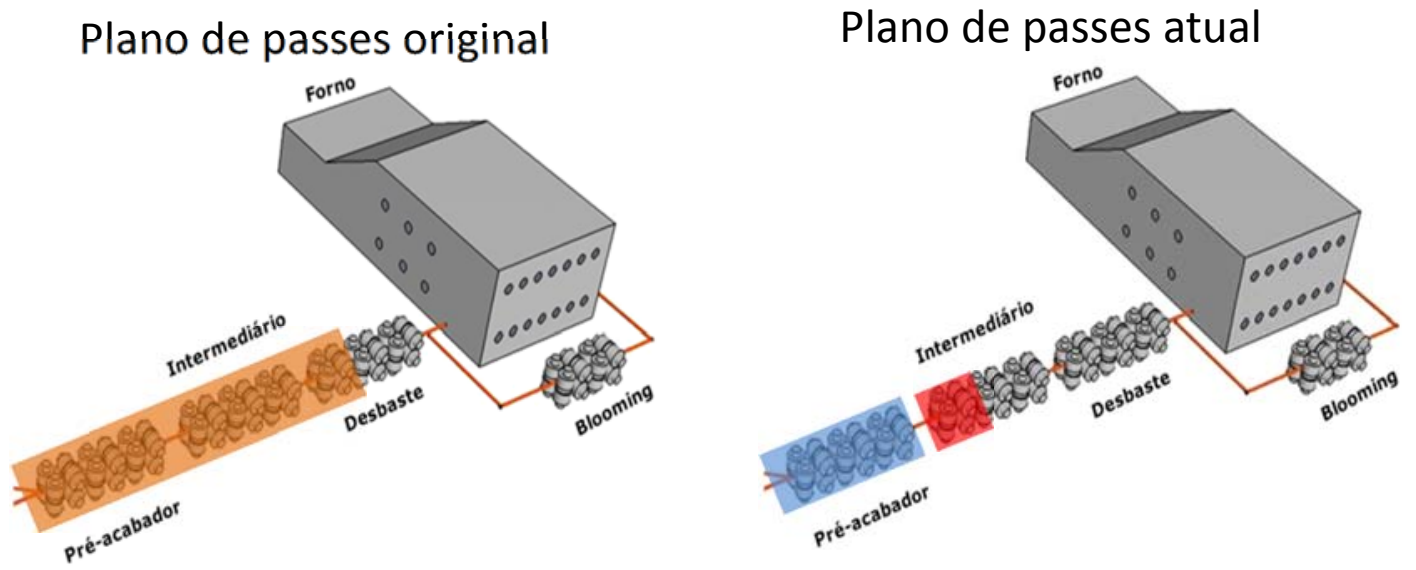

Figura 6 - Montagem esquemática do laminador. 
Após esta etapa iniciamos os testes no laminador bitola por bitola. Todas as bitolas testadas apresentaram o resultado esperado e obtido através da simulação.

\section{RESULTADOS E DISCUSSÃO}

Abaixo mostramos uma tabela com alguns dos principais ganhos alcançados com esta melhoria no laminador:

Redução do número de cilindros

Redução número de Guias

Ganho em horas no mês*

\section{8 pares}

16 guias

6 horas

* Considerando 2 câmbios por mês.

Além destas podemos ainda citar em tempo de preparação em oficina destas cadeiras, roletes, tempo e ferramentas de usinagem e etc.

Abaixo mostramos a nova arvore de calibração com as modificações, podemos observar a simplificação e unificação do trem ate a cadeira 12, ilustrando as modificações feitas. 


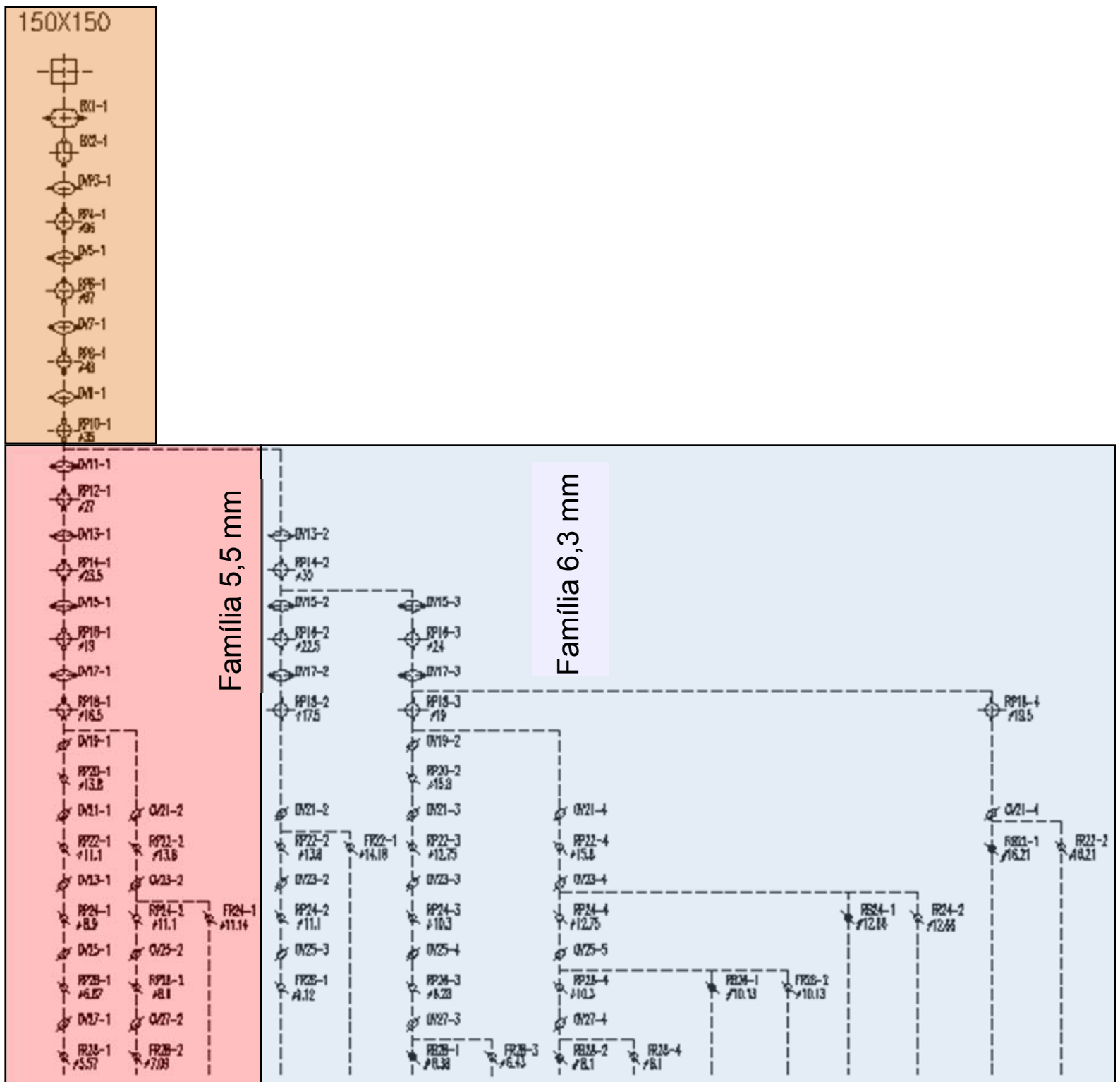

Figura 7 - Nova configuração do plano de passes. Em laranja a calibração universal, vermelho a família do 5,5 e em azul a família do 6,35.

\section{CONCLUSÃO}

As mudanças feitas no plano de passes comprovou os ganhos já esperados para este trabalho no laminador, além destes também podemos citar os ganhos na oficina de cilindros como: manutenção de cilindros, guias e simplificação nos câmbios.

Podemos concluir também que o software utilizado representou muito bem os resultados obtidos durante a produção industrial, trazendo segurança para realização dos testes por ser uma copia do ocorrido já nas primeiras etapas experimentais, levando em conta a capacidade dos equipamentos.

\section{Agradecimentos}

Aos colaboradores que ajudaram incondicionalmente o projeto em suas fases de testes e aos que incentivaram e colaboraram de forma técnica, teoria e incentivadora na execução deste trabalho. 


\section{REFERÊNCIAS}

[1] E.M.S. Rizzo - Processo de laminação de produtos longos de aço - ABM.

[2] Roberto de Cerqueira Coda - Calibração - Produtos longos de aço laminados a quente. 